\title{
Efecto de Temperatura-Tiempo Sobre los Lípidos Extraídos de Vísceras de Tilapia Roja (Oreochromis sp.) Utilizando un Proceso de Calentamiento-Congelación
}

\author{
Lorena Arias, Leidy J. Gómez y José E. Zapata \\ Grupo de Nutrición y Tecnología de Alimentos, Universidad de Antioquia, Calle 67 Número 53-108, \\ Medellín-Colombia. (e-mail: jedgar_4@yahoo.es)
}

Recibido Mar. 6, 2017; Aceptado Abr. 27, 2017; Versión final Jun. 27, 2017, Publicado Oct. 2017

\begin{abstract}
Resumen
En el presente trabajo se utilizó la metodología de superficies de respuesta para evaluar el efecto de la temperatura $\left(60-80^{\circ} \mathrm{C}\right)$ y el tiempo de calentamiento $(20-40 \mathrm{~min})$ sobre el índice de peróxido $(\mathrm{P})$, índice de yodo $(Y)$, tiempo de inducción (OSI) y concentración de grasa remanente $(\mathrm{G})$, en el proceso de recuperación de aceite de vísceras de tilapia roja (Oreochromis sp.), por medio de un método novedoso de calentamiento-congelación. Se ajustaron modelos polinomiales de segundo orden, que se optimizaron para definir los valores de los factores que entregaran la mejor combinación de las respuestas. Los resultados indicaron que las condiciones óptimas corresponden a $69^{\circ} \mathrm{C}$ y $29 \mathrm{~min}$, las cuales entregaron lípidos con $\mathrm{G}$ del 2,653\%, peróxido $\mathrm{P}$ de $0,014 \mathrm{meq} / \mathrm{kg}, \mathrm{Y}$ de $161,671 \mathrm{~g}$ yodo absorbidos $/ 100 \mathrm{~g}$ de muestra, OSI de 0,29 h y tiempo de vida útil de $808,9 \mathrm{~h}$ a $25^{\circ} \mathrm{C}$.
\end{abstract}

Palabras clave: tilapia roja (Oreochromis sp.); vida útil; tiempo de inducción; rancimat; subproductos de la piscicultura

\section{Effect of Temperature-Time on the Extracted Lipids from Viscera of Red Tilapia (Oreochromis Sp.) using a Heating- Freezing Process}

\begin{abstract}
The response surface methodology was used in this study to evaluate the effect of temperature $\left(60-80^{\circ} \mathrm{C}\right)$ and heating time (20-40 min) on the peroxide index $(\mathrm{P})$, iodine index $(\mathrm{Y})$, induction time (OSI) and concentration of remaining fat $(\mathrm{G})$, in the oil recovery process from viscera of red tilapia (Oreochromis $s p$.), through a novel method of heating-freezing. Second order polynomial models were fitted, and by optimization the values of the factors that delivered the best combination of the responses were determined. The results indicated that the optimum conditions correspond to $69^{\circ} \mathrm{C}$ and $29 \mathrm{~min}$, which gave lipids $\mathrm{G}$ $2,653 \%$, peroxide $P 0,014 \mathrm{meq} / \mathrm{kg}, \mathrm{Y}$ of $161,671 \mathrm{~g}$ of iodine absorbed $/ 100 \mathrm{~g}$ sample, OSI $0,29 \mathrm{~h}$ and a lifetime of $808,9 \mathrm{~h}$ at $25^{\circ} \mathrm{C}$.
\end{abstract}

Keywords: red tilapia (Oreochromis sp.); shelf life; Induction time; rancimat; fish farming byproducts 


\section{INTRODUCCIÓN}

El pescado está entre los productos alimenticios más comercializados en todo el mundo, pues representan más del $9 \%$ de las exportaciones agrícolas a nivel global, a su vez es una fuente muy valiosa de proteínas y nutrientes, esenciales para una nutrición equilibrada. La pesca de captura y la acuicultura suministraron al mundo 167,3 millones de toneladas de las cuales la acuicultura suministro 73,8 millones de toneladas de pescado en 2014, casi la mitad del pescado destinado a la alimentación humana y se prevía que esta proporción aumente a un $62 \%$ para el 2030 (FAO, 2016). La tilapia representó la cuarta especie más importante de agua dulce en el mundo, cuya producción mundial rondó en 2011 los 3,95 millones de toneladas (FAO, 2015). En Colombia representó el 65,21\% de la producción piscícola. En 2011 se produjeron 82.733 toneladas de tilapia, de las cuales 48.400 toneladas métricas correspondió a la tilapia roja y plateada (Esquivel et al., 2014).

Los procesos de comercialización e industrialización de tilapia para la nutrición humana utiliza del $25 \%$ al $70 \%$ como producto comestible (Vasconcelos et al., 2011). Indicando un porcentaje muy alto de generación de subproductos sólidos que comprenden vísceras, esqueletos, cabezas y escamas que no se destinan al consumo humano, tales subproductos contiene proteínas de gran calidad, aceite con ácidos grasos omega-3 de cadena larga, micronutrientes (vitamina $A$ y $D$, riboflavina y niacina) y minerales (hierro, zinc, selenio y yodo) (FAO, 2015; Martínez-Álvarez, 2011; Rai et al., 2010). Las vísceras de pescado suponen entre 7,5 $15 \%$ del peso corporal del animal y tienen un alto contenido de ácidos grasos esenciales polinsaturados (Swapna et al., 2011) como ácido docosahexaenoico (DHA) y ácido eicosapentaenoico (EPA), los cuales son altamente valorados por sus propiedades profilácticas y terapéuticas en el tratamiento de diversas situaciones nutricionales y enfermedades (Paucar-Menacho et al., 2015). Estos ácidos grasos son los principales componentes de los fosfolípidos, los cuales a su vez son las principales moléculas en la mayoría de las membranas biológicas (Swapna et al., 2010). En las vísceras de tilapia roja (Oreochromis sp.) el contenido de aceite oscila alrededor $28 \%$ (Baez et al., 2016), por lo que su recuperación constituye un aspecto relevante, ya que este residuo usualmente es descartado al ambiente generando además importantes impactos negativos (Martinez-Álvarez, 2011).

Estas características del aceite de pescado, han hecho que en los últimos 60 años pase de ser un desecho a un producto altamente valorado en la industria alimentaria (Valenzuela et al., 2012), lo cual ha generado que se desarrollen diferentes métodos para su obtención y recuperación entre los que se cuenta: hidrolisis enzimática (Swapna et al., 2011), ensilaje biológico (Rai et al., 2010), prensado en peces de descarte (PérezGálvez et al., 2011) y extracción con fluidos supercríticos (Adeoti y Hawboldt, 2015).

La mayoría de estos métodos presentan altos costos de inversión o tiempos de proceso prolongados, lo cual no ha permitido su implementación en sistemas productivos pequeños o de escala moderada. Con esto se crea la necesidad de trabajar en métodos que mejoren la eficiencia del proceso de extracción, reduzcan los costos y faciliten las condiciones de implementación en áreas rurales en diferentes escalas productivas. Un método que ha sido usado a nivel artesanal para recuperar este aceite consiste en calentar las vísceras a ebullición en recipientes metálicos durante varios minutos, para luego separarlo del resto de la masa, por sedimentación. Pero las altas temperaturas usadas en este proceso (alrededor de $90^{\circ} \mathrm{C}$ ) pueden provocar oxidación del aceite y degradación de los ácidos grasos (Fournier et al., 2006), involucrando pérdidas nutricionales, sabores indeseables y rancidez inaceptable (Navas, 2010). Por lo tanto, controlar los tiempos y temperaturas de trabajo en este proceso, así como evaluar el efecto que se pueda infringir sobre la vida útil del aceite, son aspecto de primera importancia (García-Moreno et al., 2013). Uno de los métodos más modernos para medir el grado de deterioro del aceite es el método de Rancimat (Focke et al., 2016). Este es ampliamente utilizado por ser confiable, reproducible, no demanda consumo de reactivos y las medidas pueden ser monitoreadas automáticamente a través del tiempo (Rodríguez et al., 2015; García-Moreno et al., 2013).

En el presente estudio se buscó optimizar las condiciones de temperatura-tiempo en el proceso de extracción de aceite de vísceras de tilapia roja (Oreochromis $s p$.) utilizando la metodología de superficies de respuesta para evaluar el efecto de los factores (temperatura y tiempo) sobre las variables respuesta ( $P, Y, O S I, G)$, en las vísceras desengrasadas. Así mismo se determinó la vida útil del aceite desengrasado bajo las condiciones óptimas establecidas en el diseño experimental.

\section{MATERIALES Y MÉTODOS}

La metodología se divide en varias subsecciones, donde se enuncia: la materia prima utilizada y el tratamiento al que fue sometida, los equipos experimentales empleados, la descripción de los diferentes análisis fisicoquímicos y métodos estandarizados como es el estudio de vida útil; realizados en las diferentes etapas del trabajo, el diseño experimental propuesto y el análisis estadístico usado, por último se describe el escalado del proceso. 


\section{Manejo de la materia prima}

Las vísceras de tilapia roja (Oreochromis sp.) fueron recolectadas en la piscícola El Gaitero ubicado en el municipio de Sopetrán - Colombia, transportadas en neveras de icopor con geles refrigerantes hasta el laboratorio y procesadas el mismo día para evitar la emulsificación de la muestra al ser almacenada a bajas temperaturas $\left(-18^{\circ} \mathrm{C}\right)$, lo que dificultaría la posterior separación de las fases.

\section{Análisis fisicoquímico de las vísceras y los lípidos}

Se realizó una caracterización fisicoquímica a las vísceras de tilapia roja frescas, a las vísceras de tilapia desengrasadas, al aceite extraído por calentamiento y al aceite control mediante los métodos descritos a continuación, todos los análisis se realizaron por triplicado y con las muestras descongeladas a temperatura ambiente:

La humedad se determinó empleando una balanza de humedad con lámpara halógena modelo MOC63 (Shimadzu, Suiza). La proteína con la técnica de microkjendahl (AOAC 2.057, 1984), con un equipo modelo DK 12 heating digester (Velp Scientifica, USA). El extracto etéreo se determinó de acuerdo al método oficial de extracción por solventes (AOAC 7.062,1984). Para las cenizas se calcinaron las muestras a $550^{\circ} \mathrm{C}$ en una mufla modelo D8 (Terrígeno, Colombia) (AOAC 14.006, 1984). El índice de yodo y el de peróxidos se analizaron según las normas técnicas Colombianas (NTC) 283 y 236 (ICONTEC, 2011), respectivamente.

\section{Determinación del índice de estabilidad oxidativa (OSI)}

En este método se hace pasar un flujo constante de aire a través de la muestra mientras se controla la temperatura en un nivel elevado, con el fin de acelerar la oxidación de la misma y reducir el tiempo del análisis (Villanueva et al., 2013). El proceso de oxidación es conducido por reacciones de radicales que implican las estructuras de ácidos grasos insaturados. Durante una fase de inducción inicial no se forman prácticamente ningún producto secundario. Esto es seguido abruptamente por una fase de oxidación que se caracteriza por un rápido incremento en el índice de peróxidos y la formación de productos volátiles, los cuales según la base del método, consisten en ácido fórmico (Focke et al., 2016).

En este estudio, la determinación del OSI se llevó a cabo en un equipo Rancimat modelo 892 (metrohm, Suiza). La muestra contenida en un recipiente de reacción se calentó y se hizo pasar aire continuamente a razón de $20 \mathrm{~L} / \mathrm{h}$ y $120^{\circ} \mathrm{C}$, al cabo de un tiempo los ácidos grasos se descompusieron y se formaron ácidos orgánicos volátiles que fueron trasportados por una corriente de aire hasta un vaso de medición que contenía agua destilada. Allí tuvo lugar una medición continua de conductividad eléctrica y el aumento de esta indico la presencia de ácidos orgánicos. El tiempo trascurrido hasta la formación de estos productos de oxidación es lo que se conoce como OSI (Focke et al., 2016).

\section{Determinación de vida útil}

El tiempo de estabilidad oxidativa determinado por el método Rancimat a temperaturas elevadas para un aceite en particular, se puede extrapolar a temperaturas bajas como las empleadas en almacenamiento; el tiempo hallado vendría a ser teóricamente la "vida útil" a temperatura de almacenamiento (Rodríguez et al., 2015). El método de extrapolación incorpora la cinética de reacción y se basa en un cálculo tipo Arrhenius entre los valores de OSI y la temperatura (Dinkov et al., 2015). En el presente estudio, se tomaron los valores de OSI a altas temperaturas $\left(100,120\right.$ y $\left.140^{\circ} \mathrm{C}\right)$ con flujo de aire de $20 \mathrm{~L} / \mathrm{h}$ y se extrapolaron para hacer una estimación aproximada de la caducidad de la muestra a $25^{\circ} \mathrm{C}$. La ecuación (1) describe la relación entre temperatura de almacenamiento y velocidad de degradación (Dinkov et al., 2015).

$$
\mathrm{OSI}=\mathrm{OSI} 0 \times \exp (\mathrm{B} \times 1 / \mathrm{T})
$$

Tomando $B=E a / R$ y linealizando, se llega a la ecuación (2), la cual establece la dependencia de los valores de OSI con respecto a la temperatura termodinámica y entrega la energía de activación de las reacciones de oxidación del aceite.

$$
\operatorname{Ln}(\mathrm{OSI})=\ln (\mathrm{OSI})-\frac{\mathrm{Ea}}{\mathrm{R}}\left(\frac{1}{\mathrm{~T}}\right)
$$

En esta ecuación, OSI es el índice de estabilidad oxidativa ( $h$ ); $E_{a}$ es la energía de activación ( $\left./ \mathrm{mol}\right)$; OSlo es el factor de Arrhenius (h); $R$ es la constante universal de los gases $(8,314 \mathrm{~J} / \mathrm{mol}$.K) y $T$ es la temperatura en Kelvin (K). 


\section{Extracción de aceite con calentamiento y congelación}

En este proceso los ensayos se llevaron a cabo en tubos falcon de $50 \mathrm{ml}$ los cuales se llenaron con $35 \mathrm{~g}$ de muestra y se procedió a introducirlos en un baño seco AccuBlock modelo D1200 (Labnet, USA) para controlar la temperatura del proceso. Posteriormente las muestras se dejaron atemperar hasta que la temperatura descendió a $45^{\circ} \mathrm{C}$, para luego llevarlos a congelación donde la temperatura del medio era de

$-18^{\circ} \mathrm{C}$ por $24 \mathrm{~h}$ para una mejor separación del aceite, dado que al solidificarse las fases lo hacen por separado. Pasado este tiempo se procedió a desprender la fase lipídica de la superficie aun congelada del producto por medio de un corte transversal y se dio comienzo a la realización de los análisis.

\section{Diseño de experimentos}

Para optimizar el proceso se llevaron a cabo 13 corridas experimentales por medio de un diseño experimental factorial central compuesto para evaluar el efecto de la temperatura y el tiempo de calentamiento en la separación del aceite de las vísceras, tomando como variable respuesta el índice de yodo (Y), g Yodo absorbidos /100 g de muestra, índice de peróxido (P), meq-g de O2 consumido por kg de grasa, índice de estabilidad oxidativa o tiempo de inducción en $\mathrm{h}$ por el método Rancimat (OSI) y porcentaje de aceite en las vísceras desengrasadas por el método de extracción Soxhlet $(G)$.

La metodología de superficies de respuesta asume que existe una función polinomial que relaciona las respuestas con las variables independientes en el proceso (factores), por eso los datos experimentales obtenidos del diseño (tabla 1) se ajustaron a un polinomio de la forma mostrada en la ecuación (3) (Montgomery, 1991).

$$
\text { Respuesta }=\alpha_{0}+\alpha_{1} X_{1}+\alpha_{2} X_{2}+\alpha_{11} X_{1}^{2}+\alpha_{22} X_{2}^{2}+\alpha_{12} X_{1} X_{2}
$$

En esta ecuación, los $\alpha i$, son constantes de ajuste y $X_{1}, X_{2}$, son Temperatura ( $T$ ) y tiempo (Ti) respectivamente.

En la tabla 1 se presentan los niveles de los factores, los cuales se aplicaron en el diseño experimental utilizando para ello el software Design-Expert 8.0 (Stat-Ease Inc, USA).

Tabla 1: Niveles de los factores en el diseño factorial central compuesto

\begin{tabular}{|c|c|c|c|}
\hline Factor & Unidades & Mínimo & Máximo \\
\hline Temperatura & ${ }^{\circ} \mathrm{C}$ & 60 & 80 \\
\hline Tiempo & Min & 20 & 40 \\
\hline
\end{tabular}

Se desarrolló el análisis de varianza (ANOVA) con un nivel de confianza del 95\%, el cual incluye la significancia estadística de cada uno de los términos del modelo ajustado (Valor - P), los coeficientes estimados en cada término ( $\alpha \mathrm{i})$, el coeficiente de determinación del modelo $\left(R^{2}\right)$ y la carencia de ajuste, con el fin de establecer la exactitud del modelo para representar los datos.

\section{Optimización}

Con el polinomio ajustado basado en el ANOVA, se desarrolló un proceso de optimización para determinar los niveles de los factores (T y Ti) que entregan los valores óptimos de las respuestas, como son P, Y, OSI y G. A dichas condiciones óptimas se desarrollaron tres repeticiones de los ensayos experimentales, para determinar la validez de los modelos obtenidos.

\section{Obtención del Aceite Control}

De una muestra de vísceras sin ningún procesamiento se extrajo aceite por centrifugación. Para lo cual las vísceras se centrifugaron por $15 \mathrm{~min} / 8500 \mathrm{rpm}$, a $4^{\circ} \mathrm{C}$, usando un equipo modelo U-320R (Boeco, Alemania), con el objetivo de tomarlo como aceite control para tener sus características como referencia y verificar los cambios que sufren los aceites extraídos por los diferentes procesos.

\section{Escalado del proceso}

Con el objetivo de evaluar si los resultados obtenidos a pequeña escala $(50 \mathrm{~mL})$ son válidos en un recipiente de mayor tamaño, se llevaron a cabo tres repeticiones a las condiciones óptimas, en una marmita cilíndrica, a fuego directo, sin agitación, con capacidad de 30 litros que se diseñó y fabrico en acero inoxidable 304. Cuenta con termopar tipo $\mathrm{J}$ el cual esta enlazado con un sistema de control PID. Este último permite accionar una válvula solenoide facilitando el paso de gas propano a los quemadores donde se efectúa la combustión 
del gas. Esta etapa se realiza por medio de 8 quemadores ubicados en la parte inferior del recipiente. El tren de gases está provisto de un regulador de alta presión el cual debe ser acoplado al cilindro de gas propano. Este regulador permite disminuir la presión del gas a la salida del cilindro y facilitar su combustión. En la figura 1 , se presenta el recipiente diseñado (30L) para escalar el proceso desarrollado a nivel de laboratorio (50 $\mathrm{mL}$ ) y en la tabla 7 se presentan los resultados del análisis del aceite extraído en este dispositivo.

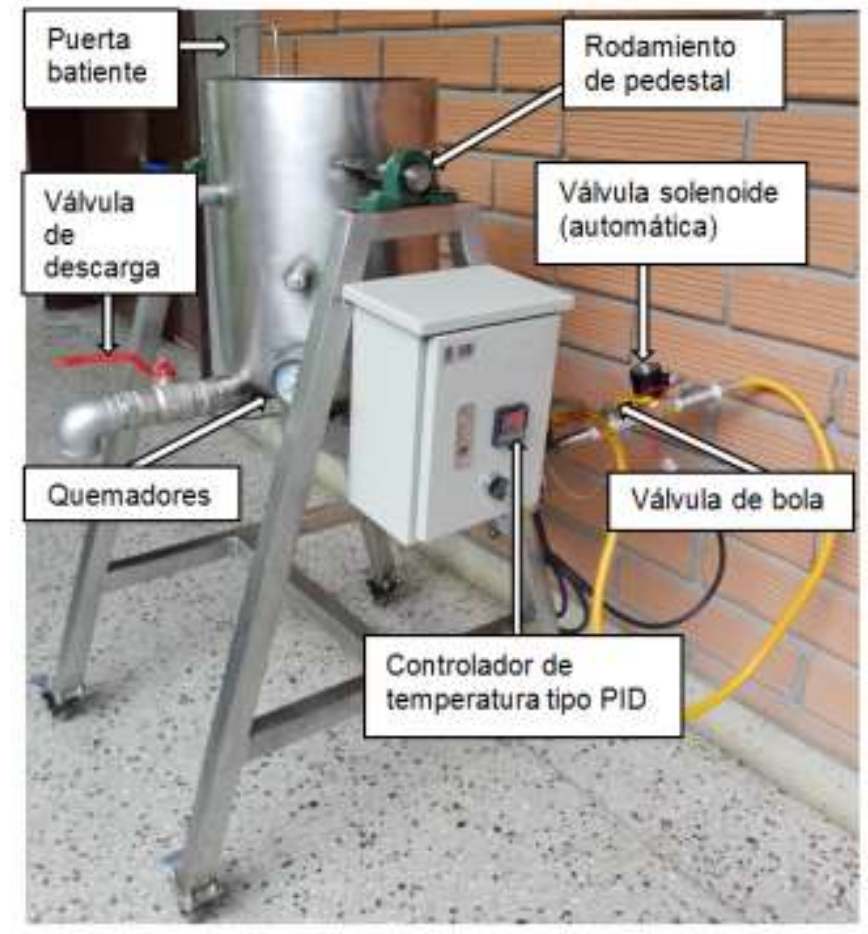

Fig. 1: Marmita cilíndrica diseñada para la extracción de aceite de vísceras de tilapia roja (Oreochromis sp.).

\section{RESULTADOS Y DISCUSIÓN}

A continuación se presenta los resultados de la caracterización de la materia prima, seguido del efecto de los factores sobre las variables respuesta y la optimización de proceso. Posteriormente se analiza la estimación de la vida útil y se finaliza con el escalado del proceso.

Caracterización del sustrato

En la tabla 2 se presenta la caracterización fisicoquímica de las vísceras, antes y después del proceso de desengrasado, en la cual se puede observar como el tratamiento aplicado reduce sustancialmente el contenido de grasa, con lo que los demás componentes incrementan también de manera significativa.

Tabla 2: Composición bromatológica de las vísceras de tilapia roja (Oreochromis sp.)

\begin{tabular}{|c|c|c|}
\hline Componente & Vísceras completas & Vísceras desengrasadas \\
\hline Grasa & $33,602 \pm 0,726$ & $2,525 \pm 0,090$ \\
\hline Humedad & $62,693 \pm 0,530$ & $82,607 \pm 0,320$ \\
\hline Proteína & $4,574 \pm 0,300$ & $12,644 \pm 1,037$ \\
\hline Cenizas & $0,732 \pm 0,380$ & $1,462 \pm 0,099$ \\
\hline
\end{tabular}

\section{Efectos de los factores sobre las respuestas}

En la tabla 3 se presentan las corridas experimentales en forma aleatoria, así como los resultados de las variables respuesta en cada una de estas. En la tabla 4, se presenta el ANOVA del diseño experimental, el que se incluye la significancia estadística del modelo ajustado (Valor - P) y el coeficiente de determinación del modelo $\left(R^{2}\right)$, mientras que en las ecuaciones 4-7, se presentan los polinomios que ajustan las respuestas en función de los factores e interacciones estadísticamente significativas, definidos en el ANOVA (tabla 4). En esta se observa que $T$ tiene efectos estadísticamente significativo sobre todas las respuestas en su término lineal, mientras que su término cuadrático tiene efecto sobre todas excepto Y. En cuanto al factor tiempo, no se tiene efecto significativo en su término lineal, pero presenta interacciones significativas de orden superior en $\mathrm{Y}, \mathrm{OSI}$ y $\mathrm{G}$. 
Tabla 3: Diseño experimental para evaluar la extracción de aceite de vísceras de tilapia roja

\begin{tabular}{|c|c|c|c|c|c|c|}
\hline Corrida & Temperatura $\left({ }^{\circ} \mathrm{C}\right)$ & Tiempo $(\mathrm{min})$ & $P$ & $Y$ & OSI & $G$ \\
\hline 1 & 70,00 & 30,00 & 0,000 & 169,465 & 0,230 & 3,376 \\
\hline 2 & 70,00 & 30,00 & 0,000 & 164,604 & 0,270 & 3,426 \\
\hline 3 & 70,00 & 30,00 & 0,000 & 167,443 & 0,275 & 4,706 \\
\hline 4 & 60,00 & 40,00 & 0,000 & 163,395 & 0,310 & 3,338 \\
\hline 5 & 70,00 & 30,00 & 0,000 & 162,697 & 0,240 & 3,498 \\
\hline 6 & 70,00 & 15,86 & 0,000 & 164,919 & 0,200 & 3,480 \\
\hline 7 & 55,86 & 30,00 & 0,000 & 170,663 & 0,560 & 11,63 \\
\hline 8 & 84,14 & 30,00 & 0,014 & 152,909 & 0,210 & 4,421 \\
\hline 9 & 70,00 & 30,00 & 0,000 & 165,423 & 0,240 & 3,446 \\
\hline 10 & 80,00 & 20,00 & 0,023 & 168,417 & 0,190 & 3,560 \\
\hline 11 & 80,00 & 40,00 & 0,025 & 169,246 & 0,180 & 3,223 \\
\hline 12 & 60,00 & 20,00 & 0,000 & 167,802 & 0,370 & 4,892 \\
\hline 13 & 70,00 & 44,14 & 0,001 & 168,044 & 0,205 & 3,607 \\
\hline
\end{tabular}

Tabla 4: ANOVA para las variables respuesta del Diseño Factorial Central Compuesto en la extracción de aceite de vísceras tilapia roja (Oreochromis sp.)

\begin{tabular}{|c|c|c|c|c|}
\hline Factor & $P$ & $Y$ & $O S I$ & $G$ \\
\hline Modelo & 0.0002 & 0.0091 & 0.0004 & 0.0347 \\
\hline $\mathrm{A}: \mathrm{T}$ & 0.0002 & 0.0006 & $<0.0001$ & 0.0077 \\
\hline $\mathrm{B}: \mathrm{Ti}$ & & 0.7855 & 0.4385 & 0.6573 \\
\hline $\mathrm{AB}$ & & 0.3029 & 0.3872 & 0.6556 \\
\hline $\mathrm{A}^{2}$ & 0.0067 & & 0.0011 & 0.0149 \\
\hline $\mathrm{B}^{2}$ & & 0.6285 & 0.0197 & 0.2817 \\
\hline $\mathrm{AB}^{2}$ & & 0.0012 & 0.0506 & 0.0543 \\
\hline $\mathrm{R}^{2}$ & 0.8186 & 0.8465 & 0.9658 & 0.8347 \\
\hline
\end{tabular}

$\operatorname{Sqrt}(P)=1.419-0.046 * T+3.723 E-004 * T^{2}$

$\operatorname{Logit}(\mathrm{Y})=\operatorname{Ln}[(\mathrm{Y}-150.00) /(171.00-\mathrm{Y})]=-139.780+2.030 * \mathrm{~T}+10.656$ * $\mathrm{Ti}-$

$0.153{ }^{*} \mathrm{~T} * \mathrm{Ti}-0.182{ }^{*} \mathrm{Ti}^{2}+2.621 \mathrm{E}-003 * \mathrm{~T}^{*} \mathrm{Ti}^{2}$

$\mathrm{OSI}=1.102-0.057 * \mathrm{~T}+0.204 * \mathrm{Ti}-2.650 \mathrm{E}-003 * \mathrm{~T} * \mathrm{Ti}+5.919 \mathrm{E}-004{ }^{*} \mathrm{~T}^{2}-3.558 \mathrm{E}-$ $003 * \mathrm{Ti}^{2}+4.624 \mathrm{E}-005 * \mathrm{~T}^{*} \mathrm{Ti}^{2}$

$$
\begin{aligned}
& \mathrm{G}=-33.182-0.701 * \mathrm{~T}+9.308 * \mathrm{Ti}-0.128 * \mathrm{~T} * \mathrm{Ti}+0.017{ }^{*} \mathrm{~T}^{2}-.159 * \mathrm{Ti}^{2}+2.189 \mathrm{E}- \\
& 003{ }^{*} \mathrm{~T} * \mathrm{Ti}^{2}
\end{aligned}
$$

El efecto de T sobre $P$, pone de manifiesto la importancia de la temperatura sobre la formación de peróxidos, puesto que tratamientos con temperaturas elevadas promueven la formación de estos compuestos, que a su vez provienen del rompimiento de enlaces dobles como se muestra en la figura 2 (Rodríguez et al., 2010). En la cual es claro que la formación de peróxidos es el resultado de la reacción entre el ácido graso y el oxígeno, $\mathrm{O}_{2}$, que ocurre cuando en laces dobles de ácidos grasos se abren para fijar dos átomos de oxígeno, reacción que es altamente favorecida por incrementos de temperatura (Rodríguez et al., 2010). 


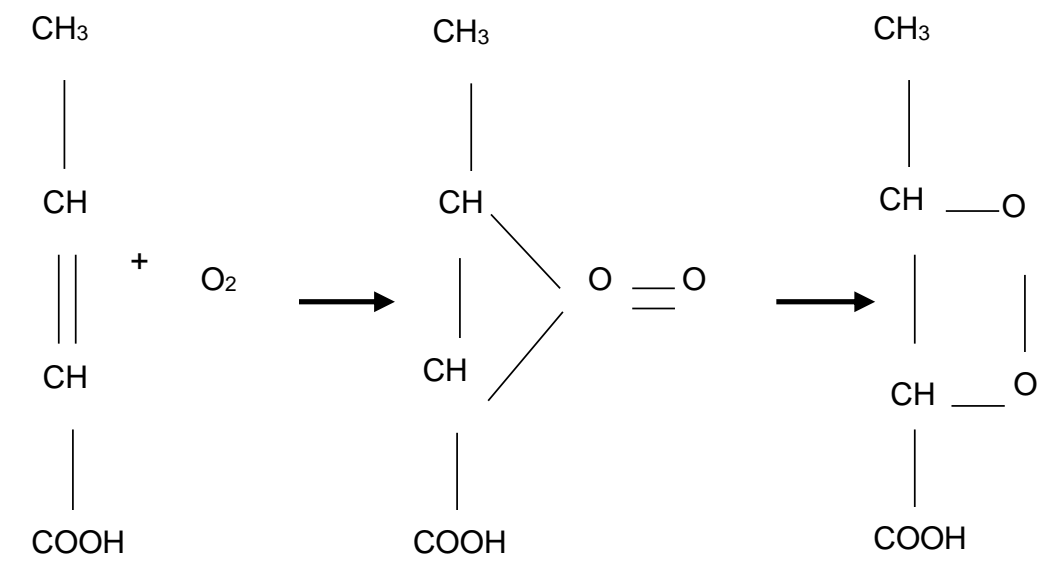

Fig. 2: Reacción de oxidación de los ácidos grasos insaturados (Rodríguez et al., 2010).

En la figura 3a, 3b y $3 c$ se observa el comportamiento gráfico de las variables $Y, O S I$ y $G$, predichas por las ecuaciones 5,6 y 7 , respectivamente. En dichas figuras se observa como las tres variables se reducen con los incrementos de $T$. En el caso de G, es conveniente que su valor se reduzca porque significa que se está recuperando una mayor cantidad de aceite, sin embargo, en el caso de OSI e Y, su reducción implica que se está perdiendo la calidad del aceite recuperado con los aumentos de T.

La figura 3a y la ecuación 5, permiten observar que existe un valor de máxima $Y$, en función del tiempo, posiblemente porque a bajos tiempos de proceso no se alcancen a liberar la mayoría de los ácidos grasos insaturados, porque permanezcan emulsificados en la matriz del sustrato, mientras que a altos tiempos de proceso pueden estarse rompiendo los dobles enlaces que son los que se detectan con el índice de yodo (Y), máxime cuando estos tiempos están combinados con temperaturas altas (Rodríguez et al., 2010), como puede observarse en la figura $3 a$ para temperaturas cercanas a $80^{\circ} \mathrm{C}$. En este punto se debe considerar que la adición de los radicales de oxígeno a los ácidos grasos se produce preferentemente en los dobles enlaces entre los átomos de carbono, siguiendo cinéticas de primer orden (Villanueva et al., 2013).

La figura $3 \mathrm{~b}$ permite apreciar de forma gráfica el efecto drástico que tiene la temperatura sobre OSI, que ya se había detectado en el ANOVA $(P<0.0001)$ (tabla 3). En dicha figura, para todos los tiempos de proceso los incrementos de temperatura reducen los valores de OSI, esto debido al efecto que la temperatura ejerce sobre la velocidad de las reacciones químicas en general (Navas, 2010) y específicamente en los procesos oxidativos en las grasas (Sathivel et al., 2008), en los cuales los incrementos de temperatura aceleran la descomposición de hidroperóxidos de lípidos (García-Moreno et al., 2013), por lo que a menores temperaturas trabajo, mayor resistencia a la oxidación, es decir valores de OSI más altos (Velasco et al., 2009).

\section{Optimización del proceso de extracción de aceites}

Las ecuaciones 4-7, se sometieron a un proceso de optimización para establecer la combinación de T y Ti, que maximizaran $\mathrm{Y}$ y OSI, y minimizaran $\mathrm{P}$ y G. En la tabla 5 se muestran los valores de las respuestas, tanto experimentales como predichas obtenidos a condiciones óptimas $\left(29 \mathrm{~min}, 67^{\circ} \mathrm{C}\right)$, en contraste con los valores del aceite control, como dato de referencia. En dicha tabla se observa que los valores experimentales están ajustados a los predichos, lo cual señala la potencia de la metodología de superficies de respuesta como método de optimización. El valor del índice de peróxidos $(P)$ experimental, es mayor que el predicho, sin embargo está muy por debajo de los valores máximos permitidos para aceites (hasta 10 miliequivalentes de oxígeno activo/kg de aceite) (CODEX STAN 19, 1981). El contenido de grasa en las vísceras extraídas (G) experimental es menor que el predicho, lo que es favorable porque se está mejorando el rendimiento de extracción. Por su parte el índice de yodo $(\mathrm{Y})$ experimental es inferior al predicho, lo que es desfavorable porque indica que se perdieron insaturaciones en el aceite durante el proceso de extracción.

En cuanto al OSI, los valores predichos y experimentales coinciden, sin embargo ambos son bastante bajos con respecto al aceite control. Estos valores de OSI son menores que los reportados para aceites vegetales como el aceite de avellana (OSI 1,39 h) (Ciemniewska-Żytkiewicz et al., 2014) y el aceite de moringa (OSI 7,237 ) (Alberca y Huanca, 2015) a $140^{\circ} \mathrm{C}$, lo cual se explica en que el presente aceite no ha sido refinado y contiene mayores insaturaciones que los aceites vegetales. 


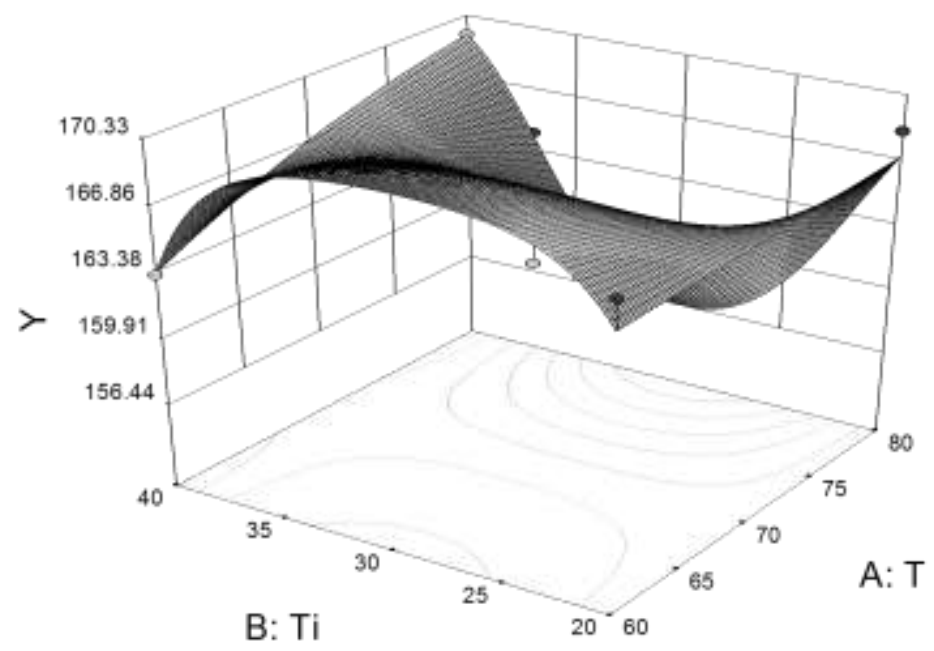

(a)

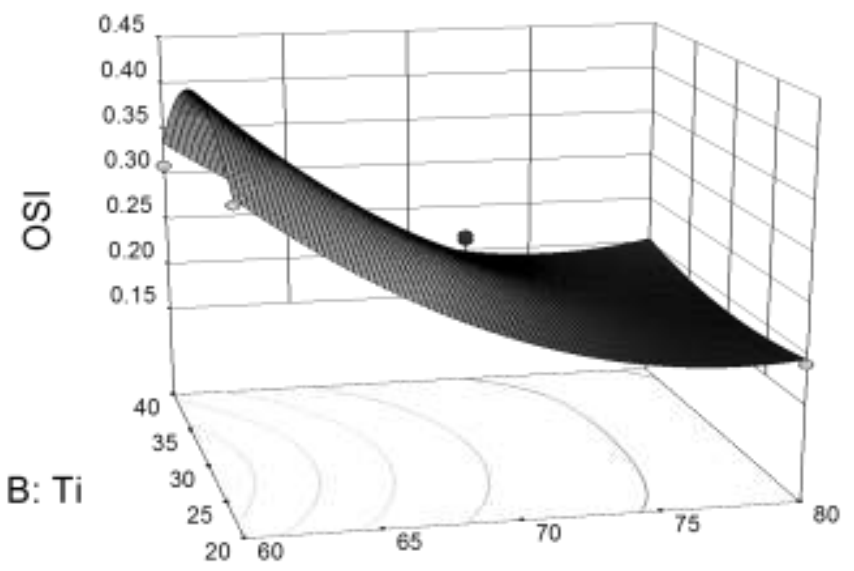

A: T

(b)

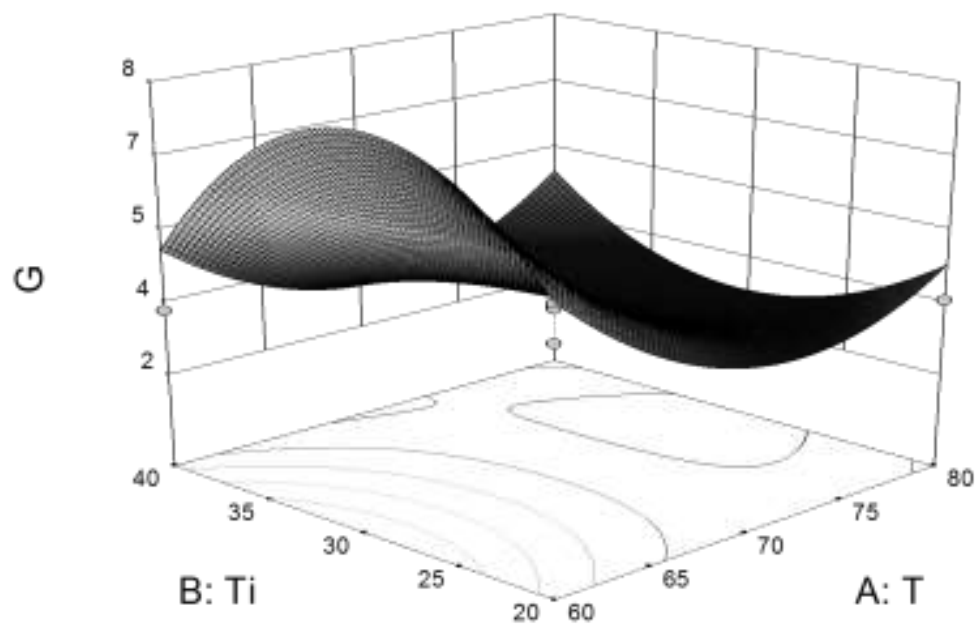

(c)

Fig. 3: Superficie de respuesta para los parámetros de $\mathrm{Y}, \mathrm{OSI}, \mathrm{G}$ en la extracción de aceite de vísceras tilapia roja (Oreochromis $s p$.) en función del T y Ti. (a) Y predicho por la ecuación 5. (b) OSI, predicho por la ecuación 6, (c) G, predicho por la ecuación 7 . 
Tabla 5: Valores experimentales y predichos obtenidos a las condiciones óptimas de extracción $\left(67^{\circ} \mathrm{C}\right.$ y 29 minutos) del aceite de vísceras de tilapia roja (Oreochromis $s p$.) comparado con el aceite control

\begin{tabular}{|c|c|c|c|c|}
\hline Valores & P & Y & OSI & G \\
\hline Predicho & 0,0 & 168,070 & 0,290 & 4,439 \\
\hline Experimental & $0,014 \pm 0,001$ & $161,671 \pm 1,304$ & $0,29 \pm 0,018$ & $2,653 \pm 0,234$ \\
\hline Aceite control & $0,004 \pm 0,002$ & $173,341 \pm 0,198$ & $0,58 \pm 0,062$ & $34,370 \pm 0,451$ \\
\hline
\end{tabular}

Estimación del Tiempo de vida útil del aceite de vísceras de tilapia roja (Oreochromis sp.)

En las figuras 4 y 5 se presenta el comportamiento de OSI en relación logarítmica con el inverso de la temperatura $\left(\mathrm{K}^{-1}\right)$ para el aceite de las vísceras procesadas y el aceite control respectivamente, mientras que en la tabla 6 aparecen los parámetros de la ecuación tipo Arrhenius, así como el $\mathrm{R}^{2}$, que indica la alta correlación existente entre las dos variables.

Tabla 6: Parámetros del ajuste tipo Arrhenius y tiempo de vida útil (OSI) a $25^{\circ} \mathrm{C}$ del aceite de vísceras de tilapia roja a $67^{\circ} \mathrm{C}$ y 29 minutos

\begin{tabular}{|c|c|c|}
\hline Coeficientes de regresión & Aceite control & $\begin{array}{c}\text { Aceite de las vísceras } \\
\text { procesadas }\end{array}$ \\
\hline $\mathrm{OSI0}$ & $1,390 \mathrm{E}-11$ & $3,597 \mathrm{E}-12$ \\
\hline $\mathrm{R}^{2}$ & 0,9521 & 0,999 \\
\hline $\mathrm{OSI}(\mathrm{h})$ & 880,100 & 808,900 \\
\hline $\mathrm{Ea}(\mathrm{kJ} / \mathrm{mol})$ & 78,866 & 82,069 \\
\hline
\end{tabular}

Es importante resaltar el alto grado correlación entre las variables, lo cual define la potencia del método utilizado. Por otro lado el descenso en la vida útil del aceite de vísceras procesadas con respecto al aceite control no es muy significativo lo que indica que el tratamiento térmico al que son sometidas las vísceras para la extracción de aceite no afecta drásticamente las características fisicoquímicas del aceite. El valor de la energía de activación es de interés en la caracterización de los aceites debido a que es indicadora de la energía necesaria para el proceso de oxidación inicial, que tiene lugar cuando se alcanza la formación de productos de oxidación primarios (Farhoosh et al., 2008). En este caso, se observa que la energía de activación del aceite control es del mismo orden de magnitud que el de las vísceras procesadas térmicamente lo que indica que el tratamiento térmico aplicado no afecta significativamente la susceptibilidad del aceite a la oxidación.

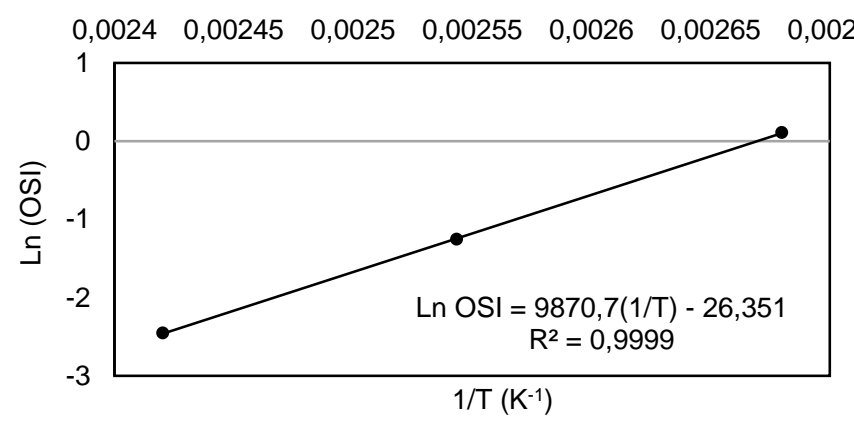

Fig. 4: logaritmo natural de OSI en función de 1/T de las vísceras extraídas con calentamiento a $67^{\circ} \mathrm{C}$ y 29 minutos.

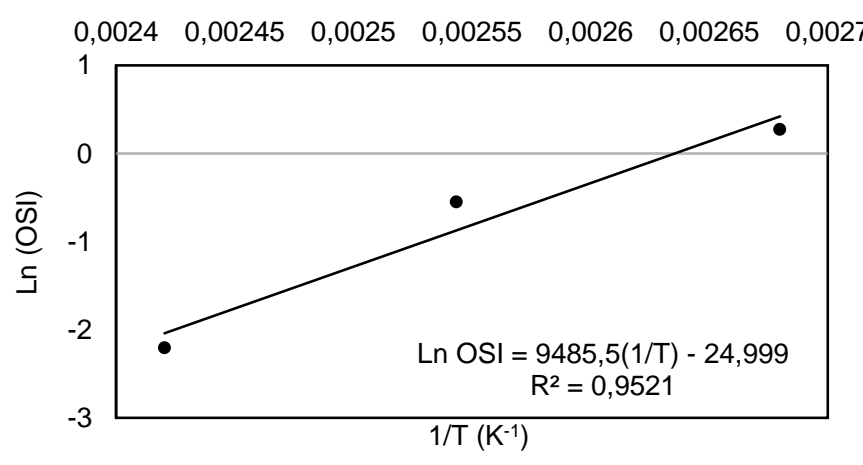

Fig. 5: logaritmo natural de OSI en función de 1/T del aceite control 


\section{Escalamiento del proceso}

Los resultados permiten ver la reproducibilidad de los valores obtenidos en las dos escalas, validando los resultados del laboratorio en un equipo que puede ser transportado a regiones apartadas, facilitando de esa forma el procesamiento de las vísceras en tiempos cortos, para reducir el deterioro del aceite.

Tabla 7: Valores experimentales obtenidos a las condiciones óptimas ( $67^{\circ} \mathrm{C}$ y 29 minutos) en el proceso de escalado de extracción de aceite de vísceras de tilapia roja (Oreochromis sp.) y parámetros del aceite control

\begin{tabular}{|c|c|c|c|c|}
\hline Valores & $P$ & $Y$ & OSI & $G$ \\
\hline Experimental & $0,016 \pm 0,011$ & $163,280 \pm 1,193$ & $0,28 \pm 0,029$ & $2,824 \pm 0,323$ \\
\hline Aceite control & $0,009 \pm 0,001$ & $171,237 \pm 0,198$ & $0,57 \pm 0,060$ & $33,602 \pm 0,098$ \\
\hline
\end{tabular}

\section{CONCLUSIONES}

(i) Es posible obtener aceite de vísceras de tilapia roja (Oreochromis sp.) que tenga características fisicoquímicas aceptable comparado con aceites de otra fuentes, utilizando el método de calentamiento congelación; (ii) La temperatura y el tiempo de la etapa de calentamiento tienen un efecto significativo sobre la cantidad y calidad del aceite extraído de este tipo de vísceras, siendo la temperatura el factor con mayor significancia; y (iii) Dicho proceso de extracción puede ser desarrollado a una escala de banco, sin afectar las características del aceite extraído.

\section{AGADECIMIENTOS}

Los autores del presente trabajo dan las gracias a la Gobernación de Antioquia, al Sistema General de Regalías de Colombia y a la estrategia de sostenibilidad 2014-2015 del Comité para el Desarrollo de la Investigación en la Universidad de Antioquia (CODI), por el apoyo financiero entregado.

\section{REFERENCIAS}

Adeoti, I. A. y K. Hawboldt, Comparison of biofuel quality of waste derived oils as a function of oil extraction methods, DOI: http://dx.doi.org/10.1016/j.fuel.2015.05.031, Fuel, (en línea), 158, 183-190 (2015)

Alberca, S. y M. Huanca, Evaluación del índice de estabilidad oxidativa del aceite de moringa (moringa oleífera) por el método Rancimat, Revista científica ingeniería: ciencia, tecnología e innovación, ISSN: 23131926 (En línea: https://goo.gl/e9N7r8, acceso 5 de septiembre de 2016), 2(2), 50-69, (2015)

AOAC, Association of Official Analytical Chemists, $14^{\text {th }}$ Ed., Washington, D.C., U.S.A. (1984)

Baez-Suarez, A. J., N. Ospina-de-Barreneche y J. E. Zapata-Montoya, Efecto de Temperatura, pH, Concentración de Sustrato y Tipo de Enzima en la Hidrólisis Enzimática de Vísceras de Tilapia Roja (Oreochromis pp.), doi: http://dx.doi.org/10.4067/S0718-07642016000600007, Información Tecnológica, (en línea), 27(6), 63-76 (2016)

Ciemniewska-Żytkiewicz, H., K. Ratusz, J. Bryś, M. Reder y P. Koczoń, Determination of the oxidative stability of hazelnut oils by PDSC and Rancimat methods. DOI: 10.1007/s10973-014-3861-9 118(2), Journal of Thermal Analysis and Calorimetry, (en línea), 118(2), 875-881 (2014)

CODEX STAN 19, Norma del Codex para Grasas y Aceites Comestibles no Regulados por Normas Individuales, 1-5, Roma, Italia (1981)

Dinkov, R. K., D.S. Stratiev, K. Shishkova, K. Ivanov, T. Saneva, M. Mitkova y M. Skumov, Assessment of shelf life of Bulgarian industrial FAME by the use of modified ASTM D2274 as accelerated oxidation method, DOI: http://dx.doi.org/10.1016/j.fuproc.2014.10.016, Fuel Processing Technology, (en línea), 130, 245-251 (2015)

Esquivel, M.A., M. C. Merino, J.J. Restrepo, A. Narváez, C.J. Polo, J. Plata y V. Puentes, Estado de la Pesca y la Acuicultura 2014. Documento de compilación de información. Autoridad Nacional de Acuicultura y Pesca -AUNAP- 26 (2014) 
FAO, El estado mundial de la pesca y la acuicultura, 274, Roma, Italia, (2015)

FAO, El estado mundial de la pesca y la acuicultura, 214, Roma, Italia (2016)

Focke, W.W., I. Van der Westhuizen y X. Oosthuysen, Biodiesel oxidative stability from Rancimat data, DOI: http://dx.doi.org/10.1016/j.tca.2016.03.023, Thermochimica Acta, (en línea) 633, 116-121 (2016)

Fournier, V.,F. Destaillats, P. Juaneda, F. Dionisi, P. Lambelet, J.L. Sebedio, O. Berdeaux, Thermal degradation of long-chain polyunsaturated fatty acids during deodorization of fish oil, DOI: 10.1002/ejlt.200500290, European Journal of Lipid Science and Technology, (en línea) 108(1), 33-42, (2006)

Farhoosh, R., R. Niazmand, M. Rezaei y M. Sarabi, Kinetic parameter determination of vegetable oil oxidation under Rancimat test conditions, doi: 10.1002/ejlt.200800004, European Journal of Lipid Science and Technology, (en línea) 110(6), 587-592 (2008)

Garcia-Moreno, P. J., R. Pérez-Gálvez, A. Guadix y E. M. Guadix, Influence of the parameters of the Rancimat test on the determination of the oxidative stability index of cod liver oil, DOI: http://dx.doi.org/10.1016/j.lwt.2012.11.002, LWT-Food Science and Technology, (en línea) 51(1), 303-308 (2013)

Martínez-Álvarez, O., Estado actual del aprovechamiento de subproductos de la industria pesquera mediante la obtención de productos de alto valor añadido, Alimentaria Revista de tecnología e higiene de los alimentos, ISSN: 0300-5755 (En línea: https://goo.gl/pFsW12, acceso 5 de septiembre 2016), 429, 71-80, (2011)

Montgomery, D., Diseño y análisis de experimentos, 3a Ed., 00- 589, Iberoamérica, México, D.C. (1991)

Navas, P., Componentes minoritarios y propiedades antioxidantes de aceites vírgenes y tortas residuales obtenidos por presión en frío a partir de fuentes vegetales convencionales y no convencionales, Tesis Doctoral, Universidad de Castilla La Mancha, Facultad de Ciencias Químicas (2010)

NTC, Grasas y aceites vegetales y animales, Bogotá, Colombia (2011)

Paucar-Menacho, L. M., R. Salvador-Reyes, J. Guillén-Sánchez, J. Capa-Robles y C. Moreno-Rojo, Estudio comparativo de las características físico-químicas del aceite de sacha inchi (Plukenetia volubilis l.), aceite de oliva (Olea europaea) y aceite crudo de pescado, DOI: 10.17268/sci.agropecu.2015.04.05, Scientia Agropecuaria, (en línea) 6(4), 279-290 (2015)

Pérez-Gálvez R., A. Guadix, M. C. Almécija, E.M. Guadix y J. P. Bergé, Response Surface Modeling of the Multiphase Juice Composition from the Compaction of Sardine Discards, DOI: 10.1007/s11947-011-0523-9, Food Bioprocess Technology, (en línea) 5(6), 2172-2182 (2011)

Rai, A.K., H.C. Swapna, N. Bhaskar, P.M. Halami, y N.M. Sachindra, Effect of fermentation ensilaging on recovery of oil from fresh water fish viscera, DOI:http://dx.doi.org/10.1016/j.enzmictec.2009.09.007, Enzyme and Microbial Technology, (en línea) 46(1), 9-13 (2010)

Rodríguez, G., E. Villanueva, P. Glorio y M. Baquerizo, Estabilidad oxidativa y estimación de la vida útil del aceite de sacha inchi (Plukenetia volubilis L.), DOI: 10.17268/sci.agropecu.2015.03.02, Scientia Agropecuaria (en línea), 6(3), 155-163 (2015)

Rodríguez S., A. Escobar y E. Ortiz, Desarrollo de una Prueba de Estabilidad Oxidativa del Aceite de Pescado, Comparando Medidas de Técnicas Bromatológicas con las de Calorimetría Diferencial de Barrido, Revista Colombiana de Física, ISSN: 0120-2650, (en línea: https://goo.gl/qxiZ9L, acceso el 29 de Agosto de 2016), 42(2), 191-195 (2010)

Sathivel S, W. Prinyawiwatkul y J. Huang, Thermal properties and applications of the Arrhenius equation for evaluating viscosity and oxidation rates of unrefined Pollock oil, DOI: http://dx.doi.org/10.1016/j.jfoodeng.2007.04.027, J. Food Eng., (en línea) 84(2), 187-932 (2008)

Swapna, H.C., A.K. Rai, N. Bhaskar y N.M. Sachindra, Lipid classes and fatty acid profi le of selected Indian freshwater fi shes, doi: 10.1007/s13197-010-0065-6, J. Food Sci. Techno. I, (en línea) 47(4), 394-400 (2010) 
Swapna, H., S.C. Hathwar, B. Bijinu, A. K. Rai y N.Bhaskar, (2011) Simultaneous Recovery of Lipids and Proteins by Enzymatic Hydrolysis of Fish Industry Waste Using Different Commercial Proteases, DOI:10.1007/s12010-010-9119-5, Applied biochemistry and biotechnology, (en línea) 164(1), 115-24 (2010)

Valenzuela, A., J. Sanhueza y F. de la Barra, El aceite de pescado: ayer un desecho industrial, hoy un producto de alto valor nutricional, DOI:http://dx.doi.org/10.4067/S071775182012000200009, Revista chilena de nutrición, (en línea) 39(2), 201-209 (2012)

Vasconcelos, M.M., M.S. Mesquita y S.P. Albuquerque, Padrõesfísico-químicos e rendimentos de silagem ácida de tilapia, Revista Brasileira de Engenharia de Pesca, ISSN: 1980-578X, 6(1), 27-37 (2011)

Velasco, J., C. Dobarganes, F. Holgado y G. Marquez-Ruiz, A follow-up oxidation study in dried microencapsulated oils under the accelerated conditions of the Rancimat test, DOI: http://dx.doi.org/10.1016/j.foodres.2008.08.012, Food Research International, (en línea) 42(1), 56-62 (2009)

Villanueva E., Castillo, D. y Rodríguez, G., Influencia de los parámetros Rancimat sobre la determinación del índice de estabilidad oxidativa del aceite de Sesamum indicum L. Scientia Agropecuaria, ISSN: 2077-9917, (en línea: https://goo.gl/GM88J1, acceso el 29 de agosto de 2016), 4(3), 173-18 (2013) 